\title{
Anti-Inflammatory and Anticoagulant Activities of Synthesized NSAID Prodrug Esters
}

\author{
Murad N. Abualhasan (D, ${ }^{1}$ Motasem Y. Al- Masri, ${ }^{2}$ Rufaida Manasara, ${ }^{1}$ Lana Yadak, \\ and Nael S. Abu-Hasan ${ }^{2}$ \\ ${ }^{1}$ Faculty of Medicine and Health Sciences, Department of Pharmacy, An-Najah National University, Nablus, State of Palestine \\ ${ }^{2}$ Faculty of Science, Department of Biology, An-Najah National University, Nablus, State of Palestine \\ Correspondence should be addressed to Murad N. Abualhasan; m_abualhasan@najah.edu
}

Received 14 March 2019; Revised 30 March 2020; Accepted 30 April 2020; Published 26 May 2020

Academic Editor: Marie-Aleth Lacaille-Dubois

Copyright ( 2020 Murad N. Abualhasan et al. This is an open access article distributed under the Creative Commons Attribution License, which permits unrestricted use, distribution, and reproduction in any medium, provided the original work is properly cited.

\begin{abstract}
Paracetamol with ibuprofen or with naproxen are frequently prescribed by doctors in combination. It was found that patients using a combination of NSAID like acetaminophen and ibuprofen experienced less pain. Patients are more compliant if these two drugs are combined in an ester form and given in one dosage form. The esterified prodrugs are hydrolyzed in humans to their active forms. In this study, two esters of paracetamol combined with ibuprofen and naproxen were synthesized as prodrugs. The physiochemical properties of these products were identified. Moreover, the bioactivities of these prodrugs were tested for its antiinflammatory and anticoagulant activities. The results showed an improved COX inhibition and anticoagulant activity compared with their parent drugs. The synthesized drugs are expected to improve patient's compliance in terms of administration frequency and will have better pharmacokinetic properties with fewer side effects.
\end{abstract}

\section{Introduction}

Esterification of drugs is very common in drug development; it has many advantages and applications; esterified drugs are used to mask bad taste, it also affects the solubility, bioavailability, and absorption and may increase the patient compliance. Many of the used antibiotics are prodrug esters, and the largest group of these prodrugs was developed to improve oral absorption and bitter tasting masking. Ester linkages of these prodrugs are usually hydrolyzed in human body by esterase enzyme that is present in plasma and various tissues. Esterase enzymes are responsible for the hydrolysis of prodrugs into their active polar water-soluble forms compared with the parent prodrug ester. A prodrug with an ester linkage can also be used to deliver two active drugs; this technique was used in the synthesis of many prodrugs that have anti-inflammatory, analgesic and antipyretic properties [1].

Ibuprofen is a nonsteroidal anti-inflammatory drugs (NSAIDs) that is used in the management of mild to moderate pain, fever, and inflammation (Figure 1).
The usual adult dose of ibuprofen is 200 or $400 \mathrm{mg}$ every 4 to 6 hours for the treatment of minor aches, mild to moderate pain, menstrual cramps, and fever. The dose may increase depending on treatment purposes such as in the case of arthritis [2]. It is present in many dosage forms including chewable tablets as well as oral suspension.

Naproxen is classified as NSAID drug used to relieve arthritis symptoms and may also be used in the treatment of mild to moderate pain, including acute gout and other painful conditions. Side effect of naproxen may include heartburn constipation and nausea [3]. The available pharmaceutical dose of naproxen tablet is $500 \mathrm{mg}$ and $250 \mathrm{mg}$. The chemical structure is shown in Figure 2.

Paracetamol (Figure 3) is a pain reliever and a fever reducer. It is used to treat many conditions such as headache, muscle aches, arthritis, backache, toothaches, colds, and fevers [4]. The most common available pharmaceutical dose of paracetamol tablet is $500 \mathrm{mg}$.

There are no reports on pharmacokinetic interaction between acetaminophen and ibuprofen or naproxen, and 


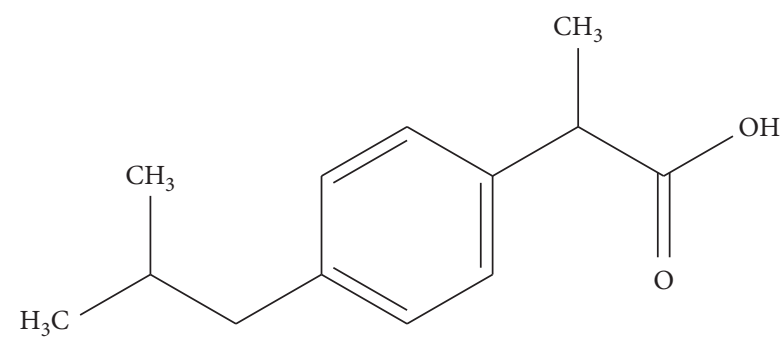

Figure 1: Ibuprofen chemical structure.

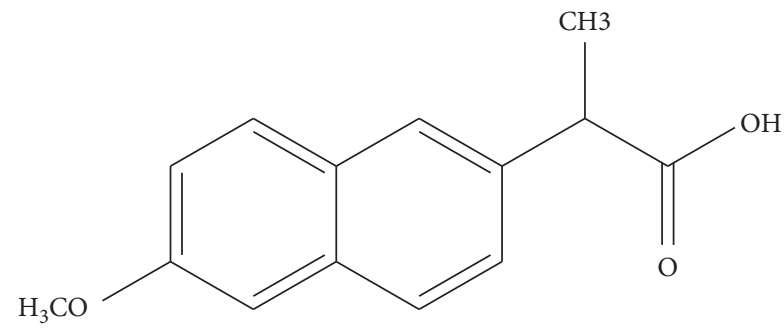

Figure 2: Naproxen chemical structure.

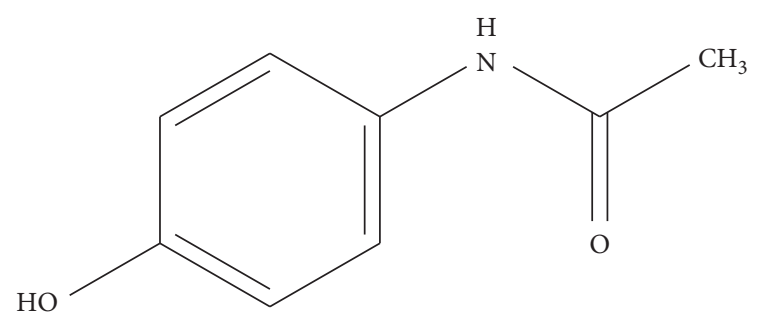

FIgUre 3: Paracetamol chemical structure.

these drugs are frequently prescribed by doctors in combination. Doctors treating pain in hospital and at home usually consider using paracetamol with other NSAIDs including ibuprofen and naproxan. Moreover, it was found that patients using the combination of NSAID like acetaminophen and ibuprofen experienced less pain during the first $48 \mathrm{~h}$ after oral surgery than those using the same daily dosage of either agent alone [5]. Consequently, it will be more convenient to patient if these two drugs are combined in an ester form and given in one dosage form.

In addition to their anti-inflammatory effects, NSAID drugs including ibuprofen were reported to have antimicrobial activity using the minimum inhibitory concentration (MIC) test $[6,7]$.

Many NSAIDs inactivate cyclooxygenase (COX) and cause platelet dysfunction by inhibiting the formation of thromboxane A2 resulting in antiplatelet effect [8]. Standard coagulation tests include the prothrombin time (PT), and activated partial thromboplastin time (aPTT) assays are general measures of extrinsic and intrinsic clotting pathway integrity, respectively $[9,10]$.

Esterification is a common chemical reaction in which two reactants, alcohol and acid, form an ester as the final reaction product [11]. Esterification can be achieved by many different synthetic pathways; some of these synthetic routes include Fischer esterification and alcoholysis of acyl chlorides with acid anhydrides. Stegliche esterification is another way of esterification that involves dicyclohexylcarbodimide (DCC) as a coupling reagent using 4-dimethylaminopyridine (DMAP) as a catalyst. The reaction was first described by Wolfgang Steglich in 1978. This reaction generally takes place at room temperature with suitable solvent like dichloromethane $[12,13]$.

The objectives of this study were to synthesize two prodrug esters that consist of ibuprofen and naproxen separately esterified with paracetamol. The synthesized prodrug esters were identified, and their physicochemical properties were determined using various techniques. Antiinflammatory and anticoagulant bioactivities of the synthesized prodrugs were also performed.

\section{Methodology}

2.1. Reagents. Paracetamol, ibuprofen, and naproxen APIs were given as a gift from Jerusalem Pharmaceutical Company- Palestine. All used reagents were of analytical grade and purchased form liable recourses; these reagents include dichloromethan (DCM), N, N'-dicyclohexylcarbodiimide (DCC), n-hexan, ethyl acetate, magnesium sulphate $\left(\mathrm{MgSO}_{4}\right)$, 4-dimethylaminopyridine (DMAP), and TLC (DC-Fertigfolien-ALUGRAM ${ }^{\circledR}$ ) silica gel (SigmaALDRICH/pore size $60 \mathrm{~A}^{\circ}, 70-320 \mathrm{mesh}$ ).

2.2. Instrumentation. The instrumentation used in this project includes magnetic stirrer and heater (LM-1003), weighing balance (Adventurer-OHAVS-AR2140), and rotaevaporator (Heidolph-OB2000). IR (infrared) spectra were done using FTIR (Thermo Fisher Nicolet IS5). The UVVIS spectra were done using the JENWAY-7315 spectrophotometer. Nuclear magnetic resonance (NMR) spectra were obtained using the Bruker Avance (300 MHz) spectrometer, micropipettes (Finnpipette, Finland), incubator (Nuve, Turkey), syringe filter $0.45 \mu \mathrm{m}$ pore size (Microlab, China), microbroth plate (Greiner bio-one, North America), and LABiTec (Germany) for PT and aPTT tests. The Cyclooxygenase (COX) enzyme inhibition test was performed using COX inhibitor screening assay kit No. 560131 Cayman Chemical, USA and was read by Elisaplate Reader (BioRad, Japan).

2.3. Chemical Synthesis. The prodrugs of NSAID-paracetamol esters were synthesized by the Steglich esterification procedure using DCC and DMAP. The reaction was performed by reacting 2 mmole of the selected NSAID, which was then dissolved in $20 \mathrm{ml}$ DCM; 1 mmole of DCC ( $0.412 \mathrm{gm})$ was added to the solution, and the mixture was allowed to stir for 24 hours. DMAP ( $40 \mathrm{mg}$ ) was then added to the mixture; the mixture was then left to stir for 24 hours. Then, 2 mmole $(0.302 \mathrm{gm})$ of paracetamol was added to the mixture, and the mixture was allowed to stir for 24 hours. The reaction was monitored by TLC using (ethyl acetate : $n$ hexane $(70: 30))$ as a mobile phase. 
Extraction and chromatographic separation of the synthesized ester was performed by the addition of $50 \mathrm{ml}$ of distilled water to the crude mixture in a reparatory funnel. The organic layer was dried using $\mathrm{MgSO}_{4}$. The organic layer was then evaporated under reduced pressure using the rotaevaporator apparatus.

The separation and purification of the drug were achieved by column chromatography. Silica gel was used as a stationary phase, and the drug was eluted using the mobile phase [ethyl acetate: $n$-hexane $(70: 30)]$. The extracted residue was dissolved with ethyl acetate and then purified using silica gel column chromatography. Proton NMR tests were performed to confirm product identification. The (NMR) spectra were obtained using the Bruker Avance $(300 \mathrm{MHz})$ spectrometer in $\mathrm{CDCl}_{3}$ using tetramethylsilane as internal standard. Chemical shifts were reported in ppm, and coupling constants were recorded in $\mathrm{Hz}$.

\subsection{Determination of Physicochemical Properties of Synthe-} sized Ester. The melting point of the solid product was determined by using a digital melting point apparatus. The sample was placed in a capillary tube and then inserted to a specific place after the instrument has been calibrated. Two temperature degrees were measured, the first one at the beginning of melting and the other one when almost all of the powder is converted to liquid.

The IR spectroscopy was determined by using an ATRFTIR. The powder was directly placed in the instrument, and the spectrum of the product was generated. UV-Vis spectrum was measured using the spectrophotometer. Approximately $1 \mathrm{mg}$ of the product was dissolved in methanol, the solution was further diluted ten times; then, the absorbance was measured in the range $200-800 \mathrm{~nm}$.

\subsection{Bioactivity Evaluation}

2.5.1. Anti-Inflammatory Effect by COX Inhibition Activity. COX1 and COX2 inhibition activities were determined using a COX inhibitor screening assay kit No. 560131 by Cayman Chemical, USA. The plate is washed with a buffer solution, and Ellman's reagent, which contains the substrate of acetylcholinesterase, is added to the well. The yellow product of this enzymatic reaction is determined spectrophotometrically in a microplate reader (BioRad, Japan) at $415 \mathrm{~nm}$. The inhibitory assays were performed in the presence of synthesized anti-inflammatory at two concentrations $(0.25$ and $0.5 \mu \mathrm{g} / \mathrm{ml})$ and compared with the commercial COX 2 selective anti-inflammatory drug such as celecoxib and for ibuprofen and naproxen, and the results were compared with those in the literature. The anti-inflammatory effect of the tested compounds was evaluated by calculating the percentage inhibition of PGE2 production. The test compound concentration causing $50 \%$ inhibition $\left(\mathrm{IC}_{50}\right)$ was calculated from the concentration-inhibition response curve by regression analysis [14].
2.5.2. Anticoagulant Activities (PT and aPTT Tests). PT and aPTT tests were carried out simultaneously. Blood samples from three healthy volunteers were collected in sodium citrated blood tubes (Vacutainer, BD). Blood samples were then centrifuged for $15 \mathrm{~min}$ at $1500 \mathrm{rpm}$ to prepare platelet poor citrated plasma. The LABiTec (Germany) apparatus was used for the determination of PT and aPTT. Equal volumes of drug samples $(3000 \mathrm{mg} / \mathrm{L})$ and platelet-depleted plasma were incubated for $5 \mathrm{~min}$ at $37^{\circ} \mathrm{C}$. The PT test was performed as follows: a $100 \mu \mathrm{l}$ of tissue thromboplastin (HUMAN) was added to $50 \mu \mathrm{l}$ of prewarmed mixture, and clotting time was measured. For the aPTT test, $50 \mu \mathrm{l}$ of rabbit brain extract was added to equal volume of the prewarmed platelet poor plasma-extract mixture incubated for 1 minute after which $50 \mu \mathrm{l}$ of $0.025 \mathrm{M}$ calcium chloride (HUMAN) was added and clotting time was measured; PBS was used as control [15].

\section{Results and Discussion}

3.1. Chemical Synthesis. Esterification reaction using Steglich esterification was successful in the formation of the intended prodrug esters. The number of protons and its chemical shift as shown in the proton NMR prove the success of the synthesis. The proton NMR of the synthesized prodrugs was found as follows:

Naproxen-Paracetamol (Supplementary Figure 3). ${ }^{1} \mathrm{H}$ NMR (300 MHz): $\delta\left(\mathrm{CDCl}_{3}\right): 1.6(2 \mathrm{H}, \mathrm{d}, J=7.24), 2.09$ $(3 \mathrm{H}, \mathrm{s}), 3.90(3 \mathrm{H}, \mathrm{s}), 4.03(1 \mathrm{H}, \mathrm{q}, J=6.89), 6.88(2 \mathrm{H}, \mathrm{d}$, 8.83), 7.11-7.74 (8H, Ar)

Ibuprofen-Paracetamol (Supplementary Figure 4). ${ }^{1} \mathrm{H}$ NMR (300 MHz): $\delta\left(\mathrm{CDCl}_{3}\right): 0.88(6 \mathrm{H}, \mathrm{d}, J=6.53), 1.57$ $(2 \mathrm{H}, \mathrm{d}, J=7.06), 2.06(3 \mathrm{H}, \mathrm{s}), 1.85$ (1H, hep), $2.06(3 \mathrm{H}$, s), $2.44(2 \mathrm{H}, \mathrm{d}, J=7.24), 3.90(1 \mathrm{H}, \mathrm{q}, 3.90), 6.78(2 \mathrm{H}, \mathrm{d}$, $J=9.01), 7.11(2 \mathrm{H}, \mathrm{d}, J=7.95), 7.26(2 \mathrm{H}, \mathrm{d}, J=7.9), 7.37$ $(2 \mathrm{H}, \mathrm{d}, J=8.83)$

The IR spectrum (infrared) of the product was obtained by the $\mathrm{KBr}$ disc technique. IR results of the synthesized and purified compound indicate that this compound is new product that probably has an ester linkage and differs from the starting material.

The IR spectrum of the prodrug product was different from the spectra of parent NSAID. The IR absorption frequencies of the functional group containing a carbonyl $(\mathrm{C}=\mathrm{O})$ in an ester has shifted, while the $\mathrm{OH}$ group of the paracetamol and the carboxylic acid of the ibuprofen and naproxen has disappeared (Supplementary Figures 1 and 2).

Spectrophotometric measurements were done in the region of ultraviolet and visible light, near-ultraviolet, and near-infrared. In the current study, wavelengths between $200 \mathrm{~nm}$ and $800 \mathrm{~nm}$ were used. The UV-Vis spectra of the prodrug esters were different from the spectra of the parent NSAIDs. The results of melting points and other physicochemical properties of the synthesized prodrugs esters are shown in Tables 1 and 2. 
TABLE 1: Physiochemical properties of synthesized naproxen-paracetamol ester.

Compound structure<smiles>COc1cccc2cc(CC(=O)Oc3ccc(NC(C)=O)cc3)ccc12</smiles>

IUPAC name

Molecular formula

Molecular weight

Melting point range

Physical characteristic

UV-Vis spectra
(S)-4-Acetamidophenyl 2-(1-methoxynaphthalen-6-yl) propanoate

$$
\mathrm{C}_{22} \mathrm{H}_{20} \mathrm{O}_{4} \mathrm{~N}
$$

$158-160^{\circ} \mathrm{C}$

Offwhite solid powder

Maximum is $230 \mathrm{~nm}$

TABLE 2: Physiochemical properties of synthesized ibuprofen-paracetamol ester.

Compound structure<smiles>CC(=O)Nc1ccc(OC(=O)C(C)c2ccc(CC(C)C)cc2)cc1</smiles>

IUPAC name

Molecular formula

Molecular weight

Melting point range

Physical characteristic

UV-vis spectra
4-Acetamidophenyl 2-(4-isobutylphenyl) propanoate

$\mathrm{C}_{12} \mathrm{H}_{25} \mathrm{NO}_{3}$

339.43

$90-95^{\circ} \mathrm{C}$

Offwhite solid powder

Maximum is $232 \mathrm{~nm}$

\subsection{Bioactivity Results}

3.2.1. COX Inhibitory Activity. The enzyme inhibition activity of the synthesized prodrugs was carried out using the ELISA kit. The $\mathrm{IC}_{50}$ of the tested compounds was calculated from percentage inhibition of COX1 and COX2 enzymes. The results showed that the calculated $\mathrm{IC}_{50}$ of the synthesized paracetamol-ibuprofen combined drug for COX1 enzyme was found to be $0.53 \mu \mathrm{g} / \mathrm{ml}$. This compound also showed an inhibition activity towards COX2 enzyme, and the calculated $\mathrm{IC}_{50}$ was found to be $2.208 \pm 0.04 \mu \mathrm{g} / \mathrm{ml}$. Paracetamol-naproxen-combined drugs showed a COX1 and COX2 inhibition $\mathrm{IC}_{50} 1.5 \pm 0.02 \mu \mathrm{g} / \mathrm{ml}$ and $1.9 \pm 0.05 \mu \mathrm{g} /$ $\mathrm{ml}$, respectively. The results clearly demonstrate that the prodrug paracetamol-ibuprofen was more selective towards COX1 compared with COX 2. In a previous published literature, ibuprofen showed inhibition activity of $1 \mu \mathrm{g} / \mathrm{ml}$ and $46 \mu \mathrm{g} / \mathrm{ml}$ for COX1 and COX2 enzymes, respectively. Naproxen inhibition activities were $2.2 \pm 0.9$ and $1.3 \pm 0.8 \mu \mathrm{g} / \mathrm{ml}$ for COX1 and COX2, respectively. Moreover, as stated in the previous literature, we found paracetamol alone has less $\mathrm{IC}_{50}$ inhibition activity for COX1 and COX2 enzymes compared with our synthesized compound. Our synthesized prodrug clearly demonstrates more inhibition activity towards COX1 and COX2 enzyme than the parent individual NSAID drugs, but it is not selective to COX2 as celecoxib $\left(\mathrm{IC}_{50}=47.5 \mu \mathrm{g} / \mathrm{ml}\right)$. This indicates that our synthesized prodrugs are expected to improve the anti-inflammatory effect compared with its parent compounds. Additionally, it expected that the patients will be more compliant to proper drug administration by taking the combined drug once rather than taking it in two separate dosage forms [15-17].

3.2.2. PT and aPTT Tests. The prothrombin time (PT) and activate partial thromboplastin time (aPTT) tests were performed for both in house synthesized esters and the parent drugs. The newly synthesized ibuprofen-paracetamol ester showed a comparable PT and PTT to the parent drug Ibuprofen as shown in Tables 3 and 4 .

The PT of ibuprofen was $(21.3 \pm 3.3)$ and for paracetamol was $(21.7 \pm 4.52)$, while for the prodrug ibuprofen-paracetamol was $(23.8 \pm 6.08)$. The PT of the synthesized naproxen-paracetamol ester was $(21.8 \pm 2.86)$. Thus, the PT was almost the same for all tested drugs.

The PTT test results showed that the prodrug ester ibuprofen-paracetamol was $71 \pm 0.9$, while the aPTT of the 
TABle 3: The PT test results.

\begin{tabular}{lcccccc}
\hline \multirow{2}{*}{ Volunteer } & & \multicolumn{3}{c}{ PT (seconds) } \\
& Normal saline & Ibuprofen & Ibu-Para ester & Paracetamol & Naproxen & Nap-Para ester \\
\hline 1 & 18.5 & 19.3 & 19.8 & 18.5 & 20 & 20.4 \\
2 & 19.4 & 19.4 & 20.8 & 19.4 & 19.5 & 19.9 \\
3 & 19.4 & 25.1 & 30.8 & 27.1 & 27.3 & 25.1 \\
Average \pm SD & $19.1 \pm 0.52$ & $21.3 \pm 3.3$ & $23.8 \pm 6.08$ & $21.7 \pm 4.52$ & $22.3 \pm 4.36$ & $21.8 \pm 2.86$ \\
\hline
\end{tabular}

TABLE 4: The aPTT test results.

\begin{tabular}{lcccccc}
\hline \multirow{2}{*}{ Volunteer } & & \multicolumn{2}{c}{ aPTT (seconds) } \\
& Normal saline & Ibuprofen & Ibu-Para ester & Paracetamol & Naproxen & Nap-Para ester \\
\hline 1 & 49 & 73.7 & 71.2 & 73.1 & 67.4 & 78.2 \\
2 & 43 & 67.6 & 71.8 & 62.8 & 66.2 & 70.9 \\
3 & 39.1 & 59 & 70 & 80.2 & 65.5 & 60.7 \\
Average \pm SD & $43.7 \pm 4.9$ & $66.8 \pm 7.3$ & $71 \pm 0.9$ & $72 \pm 8.7$ & $66.4 \pm 0.9$ & $69.9 \pm 8.79$ \\
\hline
\end{tabular}

synthesized naproxen-paracetamol ester was $69.9 \pm 7.7$ and they were also comparable to the parent drugs paracetamol and ibuprofen $[9,18,19]$.

The above result indicates that the suggested prodrugs have better COX inhibition activity compared with their parent drugs. The ester form of these drugs makes these drugs not acidic and thus will not produce primary acid insult on ulcer which is usually experienced from other commonly used NSAID. The nonselective NSAID has high incidence of peptic ulcer due to their dual peptic ulcer insult, namely, the carboxylic acid present in their structure as well as their COX inhibition effect. Moreover, the low anticoagulant effect results the synthesized compounds will probably have less bleeding side effect. Overall, these drugs as such will have different pharmacokinetic and pharmacodynamic properties before is hydrolysed to the individual NSAID which require more studies involving toxicity and side effects for the tested compounds.

\section{Conclusion}

We successfully synthesized novel combined esters of combined NSAID. The synthesized drug has improved COX inhibitory and comparable anticoagulant activities to that of the parent drugs. The combined NSAID is expected to improve the patient compliance and pharmacokinetics properties.

\section{Data Availability}

No data were used to support this study.

\section{Conflicts of Interest}

There are no conflicts of interest regarding the publication of this paper.

\section{Supplementary Materials}

This report was created by the ACD/NMR Processor Academic Edition. For more information, visit http://www.acdlabs.com/nmrproc/. (Supplementary Materials)

\section{References}

[1] S. Similä, S. Keinänen, and K. Kouvalainen, "Oral antipyretic therapy: evaluation of benorylate, an ester of acetylsalicylic acid and paracetamol," European Journal of Pediatrics, vol. 121 , no. 121 , pp. 15-20, 1975.

[2] http://www.medicinenet.com/ibuprofen/article.htm, 2015.

[3] "Summaries for patients. Gastrointestinal side effects of rofecoxib and naproxen," Annals of Internal Medicine, vol. 139, no. 7, p. 129, 2003.

[4] R. Chou and L. H. Huffman, "Medications for acute and chronic low back pain: a review of the evidence for an American Pain Society/American College of Physicians clinical practice guideline," Annals of Internal Medicine, vol. 147, no. 7, pp. 505-514, 2007.

[5] A. F. Merry, R. D. Gibbs, J. Edwards et al., "Combined acetaminophen and ibuprofen for pain relief after oral surgery in adults: a randomized controlled trial," British Journal of Anaesthesia, vol. 104, no. 1, pp. 80-88, 2010.

[6] K. T. Elvers and S. J. L. Wright, "Antibacterial activity of the anti-inflammatory compound ibuprofen," Letters in Applied Microbiology, vol. 20, no. 2, pp. 82-84, 1995.

[7] B. Forbes, D. Sahm, and A. Weissfeld, Study Guide for Bailey \& Scott's Diagnostic Microbiology, Mosby, Maryland Heights, MO, USA, 2007.

[8] A. I. Schafer, "Effects of nonsteroidal anti-inflammatory therapy on platelets," The American Journal of Medicine, vol. 106, no. 5, pp. 25S-36S, 1999.

[9] W. Z. Martini, R. Deguzman, C. M. Rodriguez et al., "Effect of Ibuprofen dose on platelet aggregation and coagulation in blood samples from pigs," Military Medicine, vol. 180, no. 3S, pp. 80-85, 2015.

[10] M. S. Park, W. Z. Martini, M. A. Dubick et al., "Thromboelastography as a better indicator of hypercoagulable state after injury than prothrombin time or activated partial thromboplastin time," The Journal of Trauma: Injury, Infection, and Critical Care, vol. 67, no. 2, pp. 266-276, 2009.

[11] IUPAC, Compendium of Chemical Terminology, IUPAC, Research Triangle Park, NC, USA, 1997.

[12] B. Neises and W. Steglich, "Simple method for the esterification of carboxylic acids," Angewandte Chemie International Edition in English, vol. 17, no. 7, pp. 522-524, 1978. 
[13] J. C. Sheehan and G. P. Hess, "A new method of forming peptide bonds," Journal of the American Chemical Society, vol. 77, no. 4, pp. 1067-1068, 1955.

[14] B. Sun Pan, Y.-Y. Kuo, T.-Y. Chen, and Y.-C. Liu, "Antioxidative and anti-inflammatory activities of two different species of a Chinese herb I-Tiao-Gung," Life Sciences, vol. 77, no. 22, pp. 2830-2839, 2005.

[15] Y. Noreen, T. Ringbom, P. Perera, H. Danielson, and L. Bohlin, "Development of a radiochemical cyclooxygenase-1 and -2 in vitro assay for identification of natural products as inhibitors of prostaglandin biosynthesis," Journal of Natural Products, vol. 61, no. 1, pp. 2-7, 1998.

[16] H.-J. Kim, Y.-H. Lee, S.-A. Im, K. Kim, and C.-K. Lee, "Cyclooxygenase inhibitors, aspirin and ibuprofen, inhibit MHC-restricted antigen presentation in dendritic cells," Immune Network, vol. 10, no. 3, pp. 92-98, 2010.

[17] J. A. Mitchell, P. Akarasereenont, C. Thiemermann, R. J. Flower, and J. R. Vane, "Selectivity of nonsteroidal antiinflammatory drugs as inhibitors of constitutive and inducible cyclooxygenase," Proceedings of the National Academy of Sciences, vol. 90, no. 24, pp. 11693-11697, 1993.

[18] M. Levine, A. D. O'Connor, A. Padilla-Jones, and R. D. Gerkin, "Comparison of prothrombin time and aspartate aminotransferase in predicting hepatotoxicity after acetaminophen overdose," Journal of Medical Toxicology, vol. 12, no. 1, pp. 100-106, 2016.

[19] E. A. J. Knijff-Dutmer, E. M. Kalsbeek-Batenburg, J. Koerts, and M. A. F. J. van de Laar, "Platelet function is inhibited by non-selective non-steroidal anti-inflammatory drugs but not by cyclo-oxygenase-2-selective inhibitors in patients with rheumatoid arthritis," Rheumatology, vol. 41, no. 4, pp. 458461, 2002. 